\title{
Analysis on the Cultural Life Situation of Local Urban Elderly in Shaanxi Province
}

\author{
Guanhua Dong \\ Xi'an University, NO1,Keji six Road, Yanta District of Xi'an, China,710065 \\ email :258332239@qq.com
}

Keywords: Community; Cultural Life; Elderly

\begin{abstract}
The cultural life of the elderly community is an important part of the community cultural life. The improvement of the quality of the elderly cultural life not only increases the satisfaction of the elderly to the society, but also has important significance for the deep development of the society. Some local urban communities in Shaanxi have problems such as the contradiction of space supply and demand, single content and imperfect management system. Therefore, the government should develop a sound safeguard system, the community cultural life environment needs to be further optimized, maintain the facilities of community cultural activities and improve the number and quality of community managers, thus helping the elderly to build a better cultural life environment.
\end{abstract}

\section{陕西地方城市老年人社区文化生活情况分析}

董冠华

西安文理学院经济管理学院, 雁塔, 西安, 陕西, 中国

email :258332239@qq.com,

关键词：社区; 文化生活; 老年人

中文摘要. 老年人社区文化生活是社区文化生活的重要组成部分, 老年人文化生活品质提升 不仅增加老年人对社会的满意度, 也对社会的深层次发展有重要意义。陕西部分地方城市社 区老年人文化生活建设存在场地供需矛盾，文化生活形式，内容单一，管理体制不健全等问 题。因此政府应制定健全的保障制度，社区文化生活环境需进一步优化，维护社区文化活动 设施以及提升社区管理人员数量和质量, 进而帮助老年人打造一个更好的文化生活环境。

\section{1. 引言}

当前人口老龄化日趋明显, 老年人有一个好的文化生活气氛在社区的发展过程中起着至 关重要的作用, 如何去提高老年人的文化生活水平、以及保护老年人身体和心理的健康, 这 对于加快社会经济结构的调整, 推动文化教育事业, 促进和谐社会的发展有着深远的意义, 怎样更好的为老年人提供一个良好的文化生活环境, 来确保他们的文化生活在当下这个社会 显得尤为重要。

\section{2. 社区老年人文化生活情况调查}

社区是进行一定的社会活动, 具有某种互动关系和共同文化维系力的人类群体及其活动 区域, 社区中人们的各种社会活动及其互动关系。为了可以准确的的客观的找出社区老年文 化生活建设中存在的问题, 我们选取了陕西省咸阳市部分社区对 100 位老年人进行了调查。 


\section{1 受教育程度}

在接受调查研究的老年人中，教育程度在小学或者小学水平以下的老年人占据了参加调 查研究的老年人总数的 $11 \%$ 、教育程度在初中水平的老年人占据了参加调查研究的老年人总 数的 $22 \%$ 、教育程度在高中或者中专水平的老年人占据了参加调查研究的老年人总数的 $29 \%$ 、 教育程度在大专或者大专以上水平的老年人占据了参加调查研究的老年人总数 的 $38 \%$ 。文化 程度的高低也影响着老年人社会功能和其他方面的差异。其中, 有文化程度的老年人相对无 文化程度的老年人来说, 具有较强的自我保健意识, 但并不是完全纯粹的正比关系, 这其中 很可能与之前的工作以及生活习惯相关，也有可能跟生活需求及目的相关。。

\section{2 生活状态}

通过调查问卷，发现老年人和其配偶两人住在一起的老年人占据了接受调查的老年人总 数的 $59 \%$ 、与配偶和子女三人(或多个儿女)住在一起的老年人占据了接受调查的老年人总数的 $37 \%$ 、老年人和儿女两个人(或多个儿女)居住的老年人占参与调研老年人总数的 $4 \%$ 。

在整个调查过程中，有无配偶的情况下，老年人的身体机能，心理状况，社会和物质功 能不尽相同, 有配偶的情况要好于无配偶的情况, 老年人在有老伴的情况下, 不仅生活质量 相对较高, 而且身心健康也相对较好。

\section{3 社区公共文化设施建设}

对所在社区公共文化设施以及服务的评价，53\%的老年人给予了肯定，认为社区在这方 面做得很不错; $21 \%$ 的人认为一般, 公共文化设施不是特别的吸引自己; 不满意或不关心的 有 $26 \%$ 。老人们比较理解居委会工作的困难, 对文化设施方面不敢提出过高的要求, 只要设 施能安全使用就基本满意了。

老年人在精神生活方面, 包含的覆盖面很广, 这其中就包括了许多社会上的活动、社会 上的活动尤指：文化精神生活，其中有唱戏、看电视、看综艺曲艺、读书、看报、电子琴、 打麻将、打牌、种菜、养花、太极拳等, 所以说, 老年人在文化生活中所需要的城市提供的 老年人文化活动中心也是非常重要的。

\section{3. 陕西地方城市社区老年人文化生活存在的问题}

\section{1 老年人文化生活所需场地的供求矛盾}

在调查的过程中, 老年人所需要许多活动场地来满足他们的文化生活需求, 但是实际上 操作的话, 没有那么简单, 居民住宅需要地方, 学校, 医院, 超市等等都会占用很多地方, 而没有那么多的空闲地供老年人作活动场所使用。

\section{2 老年人文化生活建设的形式、项目、内容单一}

老年人室内活动场所很少，平均不足 1 平米，个别社区里面没有任何基础健身设施。受访 老年人中, 有很大一部分要求增加一些运动场所或者一些文艺活动的场所来解决老年人活动 空间严重失衡的问题。目前，社区里面有一些公共报亭，少量多媒体，和娱乐设备。相对来 说, 最多的是健身设施, 社区的大部分基础设施、老年人文化活动室、阅览室等是政府扶持 建造的, 还有一部分是他们的儿女或社会上的人士支持来建造的的。现有的活动设施数量非 常有限、而且设备变得陈旧, 维护也不是很到位, 更新也相对缓慢。

\section{3 社区管理体制不健全}

\subsection{1 社区老年人文化建设动力不足}

社区的重心一般是在年轻人方面，老年人文化建设方面就相对落下了一些，再加上老年 人们自身对自己的文化生活要求可能不是很高, 积极性也相对较差, 导致社区老年人文化建 
设动力不足。随着现在人们的生存需求得到基本解决的情况后, 社区内的老年人们, 逐渐对 文化精神生活的品质提出了一些要求, 它的重要性自然凸显了出来。正因为是老年人, 所以 他们的需求和其他年龄段的人会有所不同，表现出了一些他们独特的需求，第一，老年人们 不是以功利性为首要, 而是以精神的寄托, 修养身息, 娱乐为目的的。第二, 老年人由于自 身的原因, 年龄比较大, 身体条件较差, 因此他们只能参加一些可参加的活动, 所以在整个 社会老龄化日益明显的时代下，我们不仅要满足于老年人的生活需要，也要对生活的方式给 与一定建议帮助, 最重要的是他们精神文化的建设需要进一步增强。

3.3.2 社区管理干部队伍素质偏低, 师资匮乏

如今，很多社区内部的管理人员对社区内的了解偏少。在管理方面，因为社区工作人员 大都是一些刚刚毕业的大学生, 缺少基层的工作经验, 年长的工作者人数又有限, 不能百分 之百的将自身多年来的管理经验传授给这些新来的大学生, 其次在老年人文化生活中, 缺少 一些有专业技能的老师带领老年人们进行活动, 自然就不能满足这些老年人的需求。社区管 理人员数量太少, 无法有效地进行组织, 协调、指导。

3.3.3 社区宣传不够、家庭成员的疏忽、缺乏热情以及文化活动性别差异问题

社区内对其他方面宣传的比较多，所以对老年人文化生活建设的宣传自然就变少了，而 且家庭成员没有将老年人文化生活放在一个很重要的位置上, 上心程度不够。许多社区文化 活动好不容易组织起来但参与者总是一些 “熟面孔”。参与程度不高, 就不能很好的展示社 区老年娱乐文化的特色和老年人的精神风貌, 这种现状严重影响了社区老年娱乐文化的发展。 有的社区老年人将大多数时间用在读书看报, 看电视, 只有少数老年人参加社区文化活动。 一些活动不适合女性（或男性）参与，应该充分考虑到这些问题。

\section{4 资金问题}

老年人文化建设过程中，需要很多资金来维持基础建设的维护，新器材的购置，场馆的 租赁等等一系列费用，但是政府方面能给出的资金有限。办公经费中支出及社区共建单位的 偶尔捐助，而居民自发组织的社区文化活动则主要由社区居民自筹资金。对社区文化事业的 投人严重不足，投资渠道比较单一，未能充分吸引各类经济主体参与社区文化建设，从而制 约了社区老年文化的发展。尤其是一些公益性老年文化活动缺乏稳定的资金来源，社区老年 服务事业因日常运作资金不足，无力扩大场所和添置、更新设年施。所以造成老人文化生活 建设方面进度缓慢。

\section{4. 地方城市老年人文化生活建设相关问题的建议}

\section{1 健全老年人文化生活保障制度}

政府应该积极对老龄化出现的问题进行调查研究，制定出一套完善的制度，而且还要落 实好这些政策制度, 同时, 可以考虑与每个社区的开发商进行联系, 双方就老年人文化生活 需要的一些基础设施的建设达成共识。另外在老旧社区中废弃的地方进行逐步的改造, 将闲 置资源利用起来。政府在这方面提供专款来建设, 不需要经过太多的部门, 以免造成贪污腐 败现象的滋生。在我国现今高速的发展进程中, 先进的科技基础是非常重要的, 而强盛的生 产力, 依旧需求协调稳定的环境, 这其中, 非常重要的一个环节就是老年人群体。所以老年 人文化生活建设就成了和谐的社会与老年人间的纽带, 这个过程不是一味的去创新, 去改革, 而是能找到一个平衡点, 让老年人觉得生活的很舒服, 只有这样, 才能让老年人文化生活的 发展有了良性循环，对社会的繁荣稳定发展起到帮助。

\section{2 社区文化生活环境的优化}

良好的老年人生活环境是依赖于良好的社区文化生活环境, 老年人的居住环境必须达标, 进而才能为文化生活建设打下基础, 在社区里, 应该打造出一套不独特体系, 充分利用职能 
部门对社区的主题, 个性化, 功能方向的定位等方面作出实质性的努力。其次, 我们应该壮 大我们的工作服务队伍, 让老年人自己组织老年人文化生活自管会来满足自身的需求以及相 关利益, 因为自发组织的群体广受群众尊崇, 参与到我们社区老年人自身文化建设当中来, 让他们亲身亲为的为自己社区作出贡献，这就是一种 “老有所乐，乐在其中” 的道理，这有 助于提高这些老年人自身的互助以及自助能力。另外政府应该在政策上通过一些鼓励和资金 的措施来大力支持老年人自管会的建设。帮助老年人更好的去学习新知识, 在现如今这个社 会高速发展的时代, 科学信息技术的出现, 新媒体的发展, 网络的普及, 人们的生活与以往 相比有了天壤之别, 老年人在过去根本没有多少机会去接触到这些东西, 最主要的是他们接 受这些新东西的能力差, 不能适应这些新东西, 所以在这种情况下, 就可以利用社区这个组 织, 帮助老年人通过一些活动去让他们了解一些新知识、新概念、新理念, 对信息掌握的速 度提高, 不至于太落后于现在这个高速更新化的时代, 手把手的告诉他们学习新知识的方法 途径，只有这样，才能使老年人一步步领会新知识，特别是一些中老年健康等知识。

\section{3 社区文化活动设施的更新及维护}

健身器材, 乒乓球台, 等新器材的购置, 基础设施的维护, 这些方面都应该有所加强, 以吸引老年人的参与。一个好的社区文化中，老年人不仅仅是参与者，更是体现者，一个完 整的体系是需要社区和老年人共同参与而形成的。老年人文化最重要的一部分, 社区文化可 以变得更加多元化, 而且社区文化在建设的过程中, 老年人会积极参与进来, 加强他们对社 区的认同，进而使老年人文化生活的水平提升到一个新高度。

\section{4 提升社区管理人员数量和质量}

在社区工作当中，有时候，人员数量不够会造成一些不必要的麻烦，所以社区应该在每 年的恰当时间, 招募一些大学生来社区见习或者实习，一来可以帮助社区工作人员减轻他们 的工作量, 二来还可以给大学生一个接触社会机会。当前社区管理人员对社区的基本素质和 知识面还有所欠缺, 这将会对老年人文化生活的文化环境产生影响, 所以提高社区管理人员 整体质量也显得尤为重要。

\section{References}

[1] Longfei Wang,Discussion on Cultural Construction for the Elderly in the Community, Journal of Chongqing Institute of Science and Technology, vo11, pp. 21-24, 2011.

[2] Yupeng Zhang, Self-experience and self-transcendence of older learners- A study based on self-theory of humanistic psychology, Journal of Higher Continuing Education, vo12, pp.62-67, 2013.

[3] Yinghua Tan, The Existing Problems and Solutions for the Cultural Construction of the Elderly in Zhaoqing Community - A Case Study of the Western City Community, Journal of Zhaoqing college, vol 6, pp. 11-14, 2013. 